\title{
La subjetividad de la metodología en la proyectación de la arquitectura
}

The subjectivity of the methodology in the design process of the Architecture

Pedro Arturo Martínez Osorio, ${ }^{1}$ M.Sc.

${ }^{1}$ Corporación Universitaria del Caribe - CECAR, Programa de Arquitectura. Sincelejo, Colombia. Correspondencia: pedro.martinez@cecar.edu.co

Recibido: 21 de abril de 2016. Aceptado: 23 julio 2016.

Martínez, P. (2016) La subjetividad de la metodología en la proyectación de la arquitectura. Procesos urbanos Número 3, Ene-Dic. 149-157. Doi:10.21892/2422085X.275

\section{RESUMEN}

El presente artículo presenta una reflexión sobre los procesos metodológicos utilizados en la proyectación de la Arquitectura, a partir del planteamiento de una propuesta metodológica enfocada en la investigación y aplicable al diseño arquitectónico, que fue confrontada en el año 2014, durante una experiencia de intercambio académico con la Escuela de Diseño de la Universidad Veiga de Almeida, a través de los procesos de movilidad que plantea la red Ilumno. El objetivo principal del estudio de tipo exploratorio del cual se deriva este trabajo, propone la identificación de procesos metodológicos comunes en cualquier proceso investigativo en las ciencias sociales, y su aplicabilidad a procesos metodológicos con los cuales proyectar la Arquitectura. Como resultado principal de la experiencia de contrastación de esta metodología planteada, se obtiene un pensamiento amplio con el cual abordar un problema de diseño arquitectónico. En este sentido es posible, desde la perspectiva del quehacer investigativo, entender el proceso de una manera propia, especial e inherente a cada investigador-diseñador, el cual, desde sus necesidades y procesos mentales, la apropia desde múltiples caminos hasta llegar a la respuesta más acertada como solución al problema planteado.

Palabras clave: Pedagogía, arquitectura, investigación proyectual, subjetividad

\section{ABSTRACT}

This article presents a reflection on the methodological processes used in the design process of the Architecture, based on a methodological proposal focused on research and applicable to architectural design, which was confronted in 2014, during an academic exchange experience with the School of Design of the Universidad Veiga de Almeida, through the mobility processes that the Ilumno network presents. The main objective of the exploratory study from which this work is derived, proposes the identification of methodological processes common to any research process in the social sciences and its applicability to methodological processes with which to project the Architecture. As a main result of the testing experience of this methodology, a broad thinking is obtained with which to approach a problem of architectural design. In this sense it is possible, from the perspective of the investigative task, to understand the process in a way that is unique, special and inherent to each researcher-designer, who, from his/her needs and mental processes, appropriates it from multiple paths to the more correct answer as a solution to the problem posed.

Keywords: Pedagogy, architecture, project research, subjectivity 


\section{INTRODUCCIÓN}

Este artículo es producto de la investigación denominada "Aplicación de metodologías para la investigación en el desarrollo de un objeto arquitectónico", llevado a cabo en la Corporación Universitaria del Caribe, CECAR. Se plantea en ella la relación de la arquitectura y su componente investigativo, que generó una estructura metodológica aplicable a procesos de investigación proyectación en la arquitectura, muy útil en los procesos de enseñanza y aprendizaje del diseño arquitectónico.

Este trabajo plantea una reflexión sobre la estructura de la metodología antes mencionada, a la luz de un proceso de intercambio académico dentro de la red Ilumno, con la Escuela de Diseño de la Universidad Veiga de Almeida en Rio de Janeiro, Brasil (U.V.A), en la cual se tiene la oportunidad de compartir experiencias en los procesos de proyectación dentro del laboratorio de creatividad a cargo del profesor Antônio Carlindo Câmara Lima, en U.V.A.

El laboratorio de creatividad que desarrolla la escuela de diseño de la Universidad Veiga de Almeida, es un espacio para la exploración de las distintas formas para estimular la creatividad de los alumnos de la escuela, en ese laboratorio se abordan diferentes métodos de acercamiento a los procesos creativos de proyectación del diseño.

\section{METODOLOGÍA}

La metodología con la cual se realizó el estudio de tipo exploratorio, del cual se desprende este trabajo, se desarrolló con un enfoque fenomenológico hermenéutico, en tres fases.

Una fase preliminar de tipo descriptivo, se revisa en esta la información pertinente al problema, se utilizan como instrumentos para obtener la información, la revisión documental y consultas a expertos. Como criterio base para la elección de documentos y casos de conveniencia, se establece que se trabajarán aquellos con un mayor grado de 150 Procesos accesibilidad, que registraran la tendencia en cuanto al estado de desarrollo de la temática abordada.

Una segunda fase hermenéutica, en la cual se interpretaron y analizaron los conceptos sobre el fenómeno de estudio, de tal manera que fuera posible encontrar similitudes $y$ contrastes en los discursos, con el fin de generar categorías, las cuales ayudarán a entender el problema de estudio.

Una fase final para la modelización de la metodología, en la cual, a partir de la construcción teórica, se realizó una propuesta que implica la proyección de un modelo a seguir en el cual vincular procesos investigativos en la proyectación arquitectónica, haciendo un aporte, a la visión que se tiene sobre la práctica del proceso de concepción y producción de la arquitectura, sin dejar de lado el componente artístico y creativo propio de la disciplina de la arquitectura. Es precisamente esta metodología planteada, la que es compartida y sujeta a contrastación durante el intercambio académico, la que generó la presente reflexión.

\section{La subjetividad de la metodología en la proyectación de la arquitectura.}

En muchos espacios, y sobre todo en el ámbito académico, se habla de la importancia de desarrollar métodos y procesos investigativos con el objetivo de dar rigor a los procesos de proyectación del diseño arquitectónico, son estos desde una mirada cientificista, las herramientas imprescindibles que ayudan a un arquitecto a cumplir su trabajo de una manera rigurosa, y al estudiante a formarse de una forma profesional. Uno de los autores importantes que se puede encontrar en esta discusión es Rodríguez Morales (1989), quien explica la necesidad de utilizar métodos en la proyectación de la arquitectura, por cuanto estos hacen posible el acercamiento a una respuesta más acertada y cada vez menos "prematura" o inacabada de la solución arquitectónica.

Aguilar (2000) también habla sobre el tema, a partir de su investigación denominada "Diseño y método" en la que propone una guía 
Martínez, P. - La subjetividad de la metodología en la proyectación de la arquitectura.

metodológica para ser puesta en práctica por docentes y estudiantes en busca de unificar aspectos dentro del proceso de proyectación. De igual manera plantea el proceso para desarrollar una obra arquitectónica en tres etapas: la conceptualización, la proyectación y la materialización. Estas fases comprenden los problemas arquitectónicos como problemas resueltos a través de un proceso sistémico y ordenado, el cual al ser desarrollado, tendrá recompensas al final del trabajo. Siguiendo este esquema, el arquitecto al momento de enfrentarse a un problema de diseño arquitectónico, tiene la posibilidad de abordarlo entendiéndolo como un problema de investigación, el cual desde un método riguroso estructura su trabajo, acercándose a una respuesta más acertada.

Correal (2007) habla sobre la investigación proyectual como forma de producción de conocimiento desde la arquitectura, en los siguientes términos:

La investigación proyectual comprende tres procesos fundamentales: la producción de conocimiento sobre la realidad física y material en donde se construirá el proyecto como solución, la producción de conocimiento sobre las características sociales y culturales de los sujetos y la arquitectura que demandan, y en última instancia, la investigación sobre los problemas propios de la arquitectura resueltos por medio de operaciones proyectuales construidas mediante sistemas de representación acerca de la definición de la forma y el espacio, el programa y las características técnicas de su materialización (p. 57).

En las palabras de Correal se observa una concepción sobre la investigación para los procesos de proyectación en el diseño arquitectónico, con una perspectiva muy amplia, en la cual caben aspectos relativos a la realidad física y material, a los análisis sociales y culturales, y finalmente aspectos propios del quehacer arquitectónico y su sistema de representaciones.

En atención a la necesidad de rigor solicitada en un todo proceso científico, y sabiendo que todo proceso científico parte de una pregunta la cual formula un problema, en este caso un problema de habitabilidad. La propuesta metodológica que plantea la investigación puesta en contrastación en el proceso de intercambio antes mencionado, asume como elemento clave, el método científico, tomado como el apoyo al cual debe recurrir todo investigador - diseñador que quiera dar rigurosidad a su proceso y asegurar una respuesta cercana a la mejor solución del problema (Tamayo 1999, P. 27) se considera en el análisis que se plantea, el método como el enlace que asegura la unión entre el conocimiento científico y la investigación científica.

La propuesta metodológica a partir de la investigación desarrollada en CECAR, se apoya en la tesis de que todo proceso de investigación ajustado al método científico, al ser aplicado a la arquitectura, es de gran utilidad en la práctica pedagógica del taller de diseño arquitectónico (Martínez, 2009). La utilización de procesos metodológicos de investigación en el proceso de proyectación, demanda la realización de una serie de pasos que ayudan al estudiante en la construcción ordenada de un proceso que lo llevará de forma sistemática a una solución al problema, argumentada en el análisis y reflexión de cada uno de sus componentes. Es en esta serie de pasos y sobre la rigurosidad del análisis que recae el peso en el proceso de enseñanza aprendizaje, evitando en lo posible la descalificación subjetiva por parte del "gusto" del profesor, o la improvisación despreocupada por parte del estudiante. Se abre paso a un espacio en común para la interacción docente - estudiante, el proceso de investigación proyectación, sobre el cual se realizan múltiples discusiones alrededor de los planteamientos dentro del proceso que desarrolla el estudiante en camino de solucionar el problema (ver figura1).

En cualquier proceso de investigación de manera general el investigador efectúa una serie de pasos, recorre un camino que lo lleva paso a paso hacia la respuesta más acertada al problema. Identifica, define el problema, lo plantea, es decir lo describe, analiza y formula, para luego solucionarlo, pasos que pueden ser estructurados de manera similar en problemas de habitabilidad a ser resueltos por un arquitecto. 


\section{necesidad / problema \\ एᄂ}

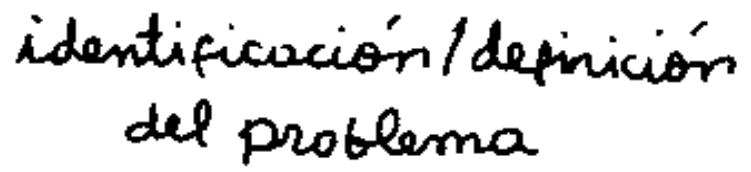

d3

Planteamiento dil proberna

il

Proyectoción

de la hipotesis

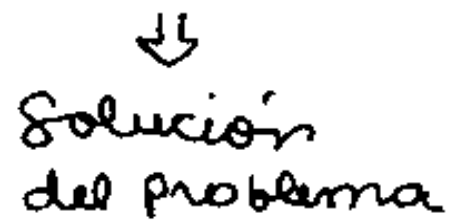

Figura 1. Estructura metodológica general de un proceso de investigación aplicable a la proyectación de la arquitectura.

Fuente: Elaboración propia

En la estructura metodológica que se propone para la proyectación en la arquitectura (ver figura 2), el arquitecto investigador asume el problema de diseño como un problema de investigación, desde el cual es necesario antes de llegar a conocer el problema, entenderlo en su complejidad, realizando una serie sistemática de pasos que conducen paulatinamente a una respuesta argumentada. De esta manera en la metodología de investigación para la proyectación en la arquitectura o metodología de investigación proyectual que se propone, el arquitecto investigador realiza pasos comunes a cualquier estructura de un proceso de investigación, partiendo de la necesidad problema, definiendo y planteando el problema, para luego formular y desarrollar una serie de hipótesis desde la proyectación, las cuales serán puestas a prueba mediante modelos a escala y validados en la obra de construcción.

Uno de los problemas de la propuesta metodológica para la investigación proyectual que se propone, es la linealidad en la representación del proceso, que puede ser algo rígido a los ojos del observador desprevenido, lo cual es un equívoco ya que se piensa que un proceso de investigación proyectual, de ninguna manera puede ser desarrollado en forma lineal sino más bien de manera cíclica, siendo posible revisarlo en cualquiera de sus fases. Por esto se plantea un esquema que pueda desvirtuar de algún modo la forma de entender esta metodología de manera lineal, sino más bien cíclica, dependiendo del camino a seguir por el diseñador (ver figura 3).

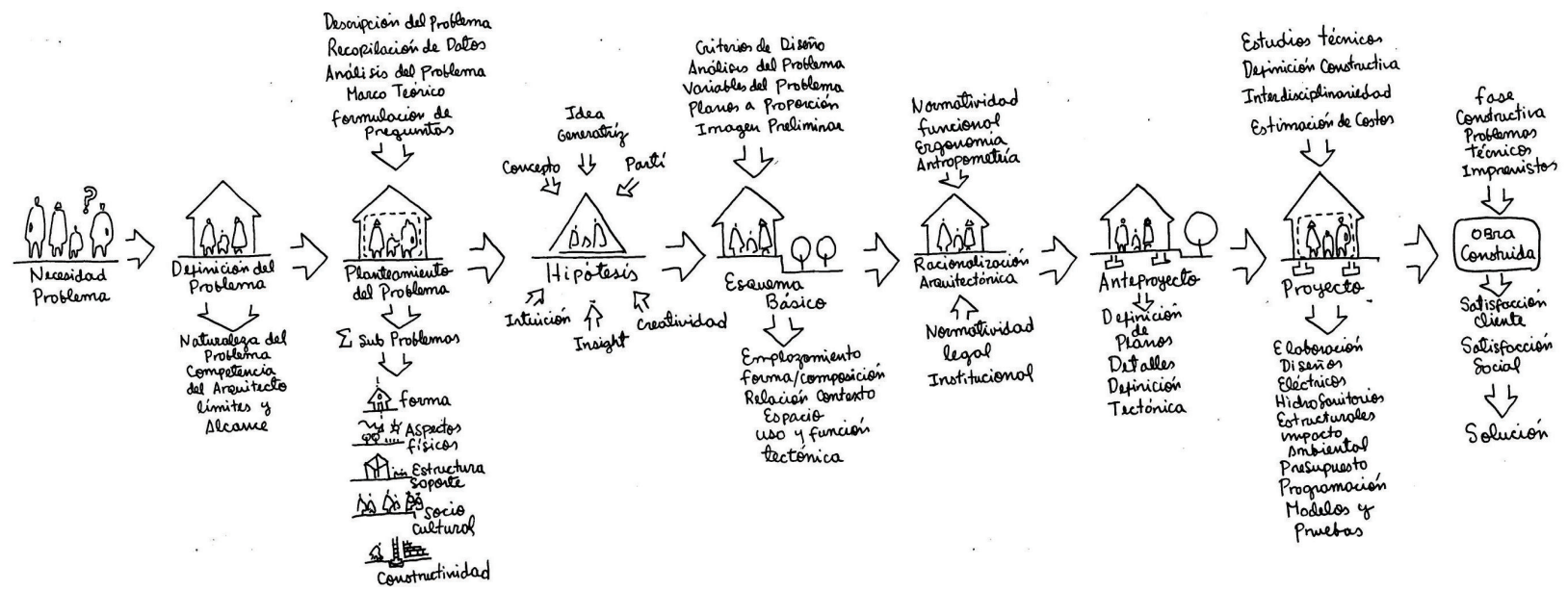

Figura 2. Estructura metodológica propuesta para ser aplicada a procesos de proyectación de la arquitectura. Fuente: Elaboración propia. 


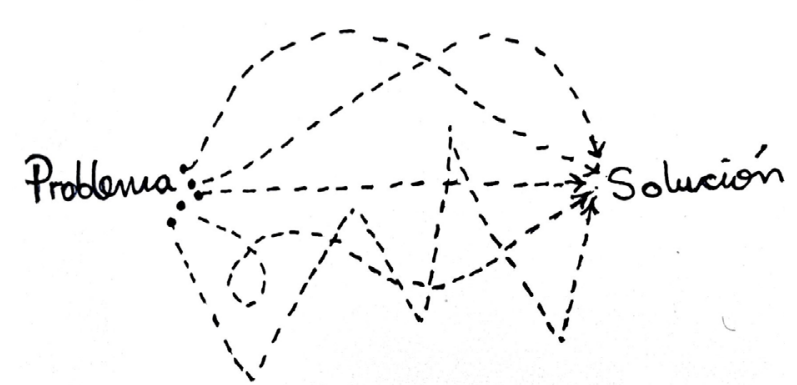

Figura 3. Diferentes caminos para llegar a la solución de un problema arquitectónico.

Fuente: Elaboración propia.

Durante la experiencia de intercambio con la escuela de diseño Universidad Veiga de Almeida en Rio de Janeiro, fue posible compartir conocimientos sobre estos procesos metodológicos para la proyectación, tanto en la arquitectura, así como para el diseño de interiores, diseño de modas o diseño gráfico, también compartir la experiencia de trabajo en el Laboratorio de creatividad de la UVA, dirigido por el profesor Antônio Carlindo Câmara Lima, quien estimula a los estudiantes con diversas metodologías que posibilitan el salto creativo en el diseñador.

Durante la experiencia en el Laboratorio, se presenta dentro de muchas posibilidades de estructurar procesos creativos en la solución de un problema de diseño la herramienta "Espinha do Peixe" o espina de pez desarrollada en 1943 por el ingeniero Kaoru Ishikawa, para facilitar el análisis de problemas y soluciones aplicadas en diferentes contextos empresariales. En el Laboratorio de creatividad, la "espina de pez" se utiliza como una estructura que hace posible reflexionar sobre un problema desde múltiples relaciones para llegar a una solución rica en reflexiones y posibilidades. Este procedimiento facilita identificar enseguida el amplio potencial de esta estructura en la representatividad de un proceso que conduce a una solución, sin ser en ningún caso de corte lineal y rígido (ver figura 4).

La herramienta "Espina de pez" al interior del Laboratorio de creatividad, examina una idea central, se detallan varias categorías o sub ideas relacionadas con el problema

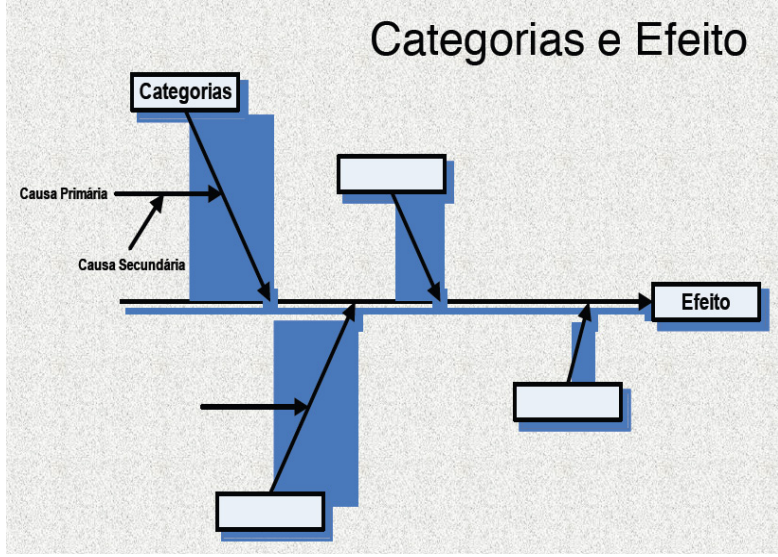

Figura 4. Adaptación de la estructura metodológica de la espina de pez. Autor prof. Antonio Carlindo Camara Lima. Laboratorio de creatividad, Universidad Veiga de Almeida.

Fuente presentación metodología Espinha do peixe, Antônio Carlindo Câmara.

fundamental. La metodología es usada registrando la idea central en la cabeza del pez, anotando las principales categorías en las espinas, especificando informaciones importantes a manera de palabras clave para hacer relaciones entre conceptos.

En el Laboratorio, el estudiante utilizando la estructura metodológica se acerca a la solución, teniendo la oportunidad de reflexionar en el proceso sobre aspectos y conceptos fundamentales para la solución. Se piensa muy positivo el hecho de poder adaptar esa estructura no lineal a la manera de representar la metodología de investigación proyectual desarrollada.

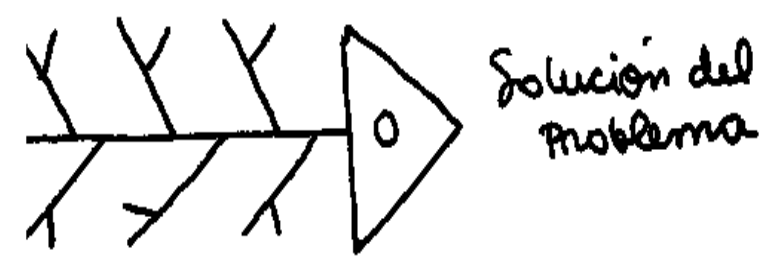

Figura 5. Representación de la estructura Espina de pez, Pedro Martínez 0. 27 - 05 - 2014.

Fuente: Elaboración propia.

También fue posible compartir la experiencia del proceso metodológico para la investigación proyectual, con distintos profesores e investigadores de la escuela de diseño de UVA, quienes encuentran positivo el proceso de estructuración de la 
metodología proyectual que se les propone, pero es importante destacar que cada diseñador, desde los procesos cognitivos que maneja y los pre saberes con los cuales afronta el problema, aborda esta o cualquier metodología desde su propia subjetividad, con lo que surge un sin número de posibilidades de representación a un proceso metodológico basado en la investigación, que tiene por objetivo desarrollar desde la proyectación, una respuesta acertada a un problema de habitabilidad.

Es posible trazar diferentes caminos para recorrer los pasos que plantea la metodología propuesta (ver figura 5). Cada diseñador la representa desde sus pre-saberes y necesidades, ya sea de forma lineal, ramificada, radial, espiral o cualquier estructura organizativa que pueda representar la manera de abordar el proceso metodológico. Es importante reflexionar en la práctica de ese proceso, sobre los elementos fundamentales, que presentados desde el enfoque de la investigación, relacionan cualquier esquema organizativo con la estructura metodológica para un proceso investigativo.

Cada diseñador tiene una visión y un método propio para abordar el problema (ver figura 6), es interesante ver la posibilidad de representarlo en cada uno y lograr adaptaciones de cada estructura, de acuerdo con los intereses y necesidades del problema. En este sentido, la metodología es una estructura flexible que no limita, al contrario, potencializa en cada investigador la seguridad de un proceso recorrido con anterioridad; también hace posibles múltiples adaptaciones de otras metodologías de acuerdo con la naturaleza del problema. En el caso de la proyectación en arquitectura, el enfoque hacia la investigación aporta un rigor necesario al proceso, lo cual potencializa la libertad creativa del diseñador, invitándolo a reflexionar sobre temas puntuales del problema a resolver.

Es por eso que en relación con las metodologías para la proyectación en la arquitectura, juega un papel fundamental el sujeto y su modo de pensar y sentir, sus pre-conceptos y la estructura trazada que ha aprehendido para solucionar los problemas del diseño. En otras palabras, en cuanto a metodologías para la proyectación del diseño es fundamental la subjetividad del diseñador. Esto último es importante en la formación de los futuros arquitectos, ya que en el camino a convertirse en profesionales, los estudiantes se enfrentarán con múltiples metodologías para acercarse a la solución del problema; es deber del docente orientador ayudarlos a estructurar una metodología propia con la cual se sientan cómodos y que sinteticen los pasos que les ayuden a reflexionar sobre temas fundamentales en el problema, antes de darle solución.

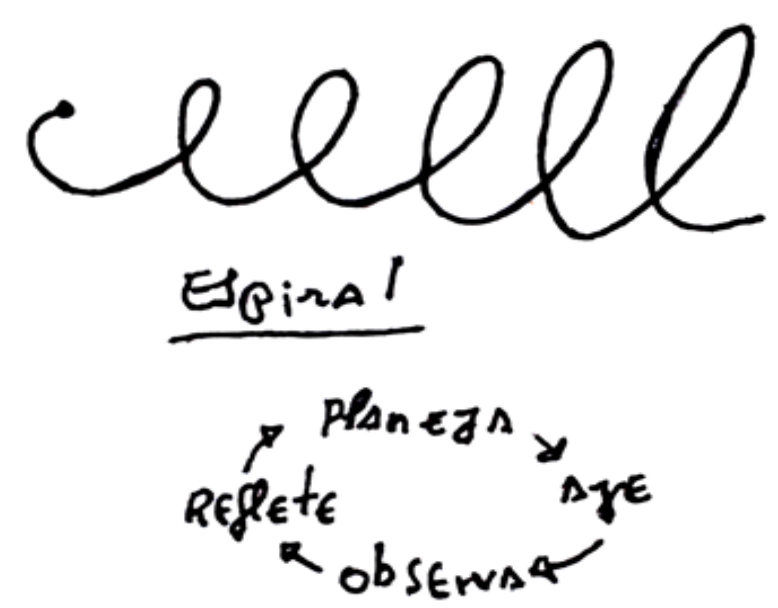

Figura 6. Reflexión del diseñador Marcos Oliva al respecto de los procesos metodológicos para la proyectación, Universidad Veiga de Almeida 27 - 05 2014.

Fuente: Elaboración propia.

\section{Discusión.}

Laenseñanza del diseñoencualquiercontexto, como camino a la profesionalización de un estudiante en los aspectos disciplinares, requiere la utilización de metodologías para abordar el problema y llegar de una forma más segura y argumentada a la solución. La perspectiva de la investigación aplicada a las metodologías de proyectación, invita a recorrer un camino sobre unos tópicos muy precisos, que más que restringir la creatividad, hacen posible que el diseñador tenga la oportunidad de reflexionar sobre aspectos claves en camino a solucionar el problema. 
Martínez, P. - La subjetividad de la metodología en la proyectación de la arquitectura.

El docente al orientar un Taller de diseño requiere el conocimiento de diversas metodologías para la proyectación, en este caso es fundamental el manejo de metodologías enfocadas en la investigación, con las cuales ofrecer al estudiante en formación diversas alternativas para acometer un problema de diseño, es útil en cualquier momento del proceso plantear al estudiante interrogantes sobre la manera de relacionar su proceso metodológico con procesos para formalizar sus productos como resultados de investigación.

La metodología de investigación proyectual es uno de tantos caminos para llegar al diseño, el cual en cuanto a la mejora de procesos desde la pedagogía, trasciende una práctica tradicionalista, para convertir al docente en un investigador y orientador del aprendizaje de la investigación en el aula, una clase especial de profesionalidad, así como es planteada por Stenhouse (citado por Galeano, 2009. P. 74), cuando habla de la enseñanza basada en la investigación.

La enseñanza de la creatividad no está desligada de la enseñanza científica como muchos pueden pensar; son aspectos ineludibles en un mundo complejo como el que sobrevivimos hoy, en el cual se necesita de miradas complejas sobre la realidad, que más que encasillarse en un paradigma, puedan abrirse a diferentes alternativas y tener una visión amplia sobre el problema y las maneras de acercarse a él, en ciertos casos "tomando distancia de lo conocido" (Fajardo y Wagner, 2003. P. 49). En ese sentido una metodología de investigación proyectual enriquece el proceso debido a que plantea interrogantes, más aún cuando casi siempre se pide al estudiante que dé respuestas a los interrogantes que plantea el docente. Fajardo y Wagner hablan sobre este tema, dando importancia al valor de la posibilidad del descubrimiento a partir de las preguntas en los siguientes términos:

"Fuimos enseñados a buscar respuestas, de preferencia acertadas, para nuestras acciones pedagógicas y así los profesores llegan con muchas expectativas para los cursos de formación. Antes que respuestas para acciones, que por lo demás no existen, sería bueno investigar nuestras intenciones, $y$ allí las preguntas son cruciales. En vez de querer "llevar al alumno a", sea donde sea, podemos indagar qué nos lleva a aprender, a practicar lo que escogemos.

La posición de alguien que se dispone a preguntar, para sí mismo y para otras fuentes de conocimiento, es extremadamente valiosa. Presupone, sin embargo, una curiosidad que no se enfoca en el acierto o el error, sino en la posibilidad del descubrimiento. Lo que el profesor ya sabe puede ser revisado, guiado por marcos de referencia establecidos por los contenidos del curso de formación, con una mirada de discernimiento desprovista de miedo al fracaso o al ridículo, así como de la necesidad de aplauso o reconfirmación por parte de una autoridad exterior". (Fajardo y Wagner, 2003. P. 50)

Para esta transición, es necesario también lograr un nuevo papel para el profesor universitario, sobre todo para los docentes arquitectos (arquitecto - investigador orientador) en un mundo dinámico como el de hoy, donde las disciplinas evolucionan con gran rapidez, y es necesaria la actualización permanente que hace posible la investigación, José Ramiro Galeano nos dice al respecto que:

"Los nuevos profesores universitarios deberán ser muy conscientes de los fundamentos propios de sus disciplinas, de la aplicación de métodos como vehículo y mapa justo del territorio que quieren explorar. Las instituciones de educación superior deben decidir si formar profesionales dentro de teorías generales o formar líderes y profesionales suficientemente autónomos para crearse sus propios espacios y contribuir imaginativa y eficazmente a la solución de las necesidades sociales; profesionales que hayan aprendido a aprender; profesionales investigadores de su propia práctica, de su campo de trabajo, siempre listos para atender los nuevos problemas y explorar las nuevas posibilidades". (Galeano, 2009. P. 75) 
Los docentes para orientar Taller de diseño se encuentran ante un nuevo reto, y es el hecho de trascender la labor docente en correspondencia con su responsabilidad social, dadas las diversas problemáticas que vivimos en el mundo de hoy, en el cual un conocimiento estático no es pertinente teniendo en cuenta las dinámicas del conocimiento en la actualidad. Es necesario evolucionar a partir de una práctica tradicionalista de Taller de diseño, ensimismado en una reproducción de las maneras de proyectar y entender la arquitectura en la cual la visión del docente es protagonista (Correal y Verdugo, 2011. P. 89), y proyectarse hacia la construcción de nuevos conocimientos tanto del docente, como del educando que asume un papel protagónico en su proceso de aprendizaje. Por lo cual la estructura de un proceso de investigación proyectual capaz de ser flexible y dinámico, es un medio para alcanzar las competencias.

Al orientar en Taller de diseño, el docente plantea una estructura metodológica para resolver el problema (ya sea lineal u otra clase de representación en su esquema), en este caso una investigación proyectual, que, desde el enfoque de la investigación, lleve al estudiante sistemáticamente a una solución argumentada al problema. Debido a la subjetividad del diseñador y para el caso la subjetividad del estudiante en el taller de diseño, el docente debe tener la capacidad de entender esa subjetividad y ayudar al estudiante en su proceso de acercamiento al problema desde la metodología, y más que limitarlo, ayudarlo a encontrar su propio camino en el cual durante el proceso se le invite a reflexionar sobre los aspectos fundamentales de problema e invitarlo a organizar su proceso de una manera coherente que le sirva para replicarla en su vida profesional.

\section{Conclusiones}

Al hablar sobre la investigación acerca de metodologías de investigación para la proyectación, se hace referencia también así sea indirectamente, a la discusión disciplinar entre arte y ciencia, la cual es un debate dinámico en el que no es posible aún hablar de una última palabra.
Cada sujeto aprehende los procesos y métodos de acercamiento a la solución de sus problemas a partir de su personalidad, preconceptos e intereses. En la formación dentro de Taller de diseño, al utilizar una metodología de investigación proyectual, se debe estar dispuesto a tener mente abierta a la adaptación del proceso por parte del estudiante, lo cual es significativo en el proceso de aprendizaje, ya que esto significa que el estudiante hace suyos unos instrumentos que el docente le plantea.

La estructura de la metodología para la investigación proyectual, que para algunos puede pensarse como un proceso rígido y demasiado lineal, es en la práctica un proceso flexible, adaptable a las necesidades y realidades dentro del proceso que aborda el diseñador, es por eso que se propone a manera de juego creativo para la misma estructura la analogía de "la hoja", una nueva manera de representar el proceso metodológico, en donde el estudiante parte de la pregunta de investigación identificando y definiendo un problema, en su camino se interroga con respecto a unos aspectos importantes dentro del planteamiento del problema, formula preguntas e hipótesis que lo llevan a seguir o a recomenzar, para llegar paulatinamente, mediante la proyectación, a la solución, la cual antes de la obra construida también se puede considerar como el punto de partida para nuevos interrogantes (ver figura 7).

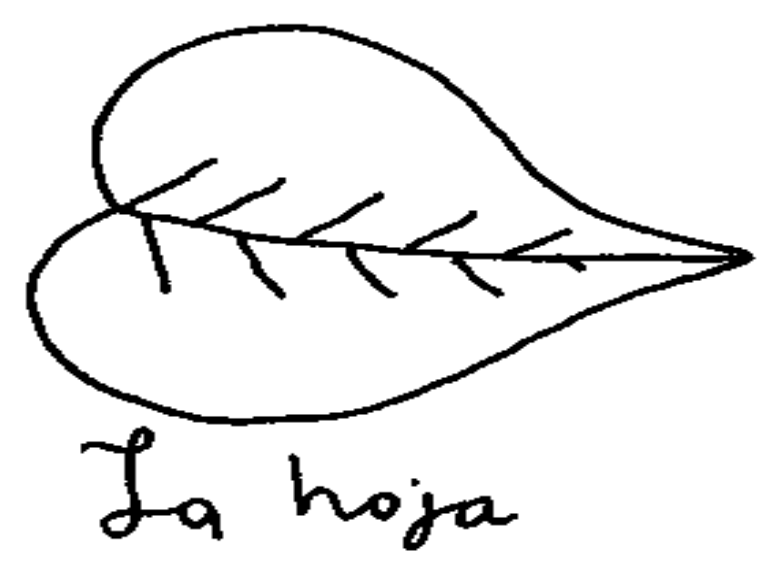

Figura 7. Posible esquema de presentación de la metodología de investigación proyectual a partir de la reflexión en el Laboratorio de Creatividad UVA.

Fuente: Elaboración propia 2014. 


\section{Referencias}

Aguilar, M. (2000). Camino al diseño, proceso del diseño arquitectónico. Manizales, Colombia. Universidad Nacional de Colombia.

Correal Pachón, G., D. y Verdugo Reyes, H. (2011, Enero - Diciembre). Sobre modelos pedagógicos y el aprendizaje del proyecto arquitectónico. Revista de Arquitectura 13, p.80 -91. Bogotá, Colombia. Universidad Católica de Colombia.

Fajardo, V. y Wagner, T. (2003) Métodos, contenidos y enseñanza de las artes en América Latina y el Caribe. División de Arte e Iniciativas Culturales del Sector de Cultura, UNESCO.

Galeano, J. (2009). Innovar en el currículo universitario. Medellín: Universidad de Antioquia.

Martínez, P. (2009). La arquitectura como problema. Sincelejo, Colombia. Corporación Universitaria del Caribe, CECAR.

Rodríguez, L. (1989). Para una teoría del diseño, México: Trillas.

Tamayo, M. (1999). Serie aprender a investigar, modulo 2, Bogotá: ICFES. 